\title{
Ericksonian hypnosis in tinnitus therapy: effects of a 28-day inpatient multimodal treatment concept measured by Tinnitus- Questionnaire and Health Survey SF-36
}

\author{
U. H. Ross $\cdot$ O. Lange $\cdot$ J. Unterrainer $\cdot$ R. Laszig
}

Published online: 15 March 2007

(C) Springer-Verlag 2007

Erratum to: Eur Arch Otorhinolaryngol

DOI 10.1007/s00405-006-0221-9

Table 1 unfortunately contained minor errors regarding the references in brackets. The correct version is shown below.

The online version of the original article can be found at http://dx.doi.org/10.1007/s00405-006-0221-9.

U. H. Ross $(\bowtie)$

Practice for Otorhinolaryngology and Psychotherapy,

Luisenstrasse 6, 79098 Freiburg, Germany

e-mail: Dr-Ross@web.de

O. Lange

Institute of Environmental Medicine and Hospital Epidemiology,

University of Freiburg, Freiburg, Germany

J. Unterrainer

Department of Neuropsychology,

University of Freiburg, Freiburg, Germany

R. Laszig

Department of Otorhinolaryngology,

University of Freiburg, Freiburg, Germany 
Table 1 Components of the treatment concept

\begin{tabular}{lll}
\hline Procedure & Sessions & Total time \\
\hline $\begin{array}{l}\text { Education } \\
\begin{array}{l}\text { Tinnitus etiology and maintenance, bio-psycho-social } \\
\text { concept of salutogenesis, sleep }\end{array}\end{array}$ & $\begin{array}{l}6 \times 90 \mathrm{~min} \\
(\text { group })\end{array}$ & $540 \mathrm{~min}$ \\
$\begin{array}{l}\text { Ericksonian Hypnosis } \\
\text { - therapeutic metaphors and symbolization [7] }\end{array}$ & $\begin{array}{l}4 \times 90 \mathrm{~min} / \mathrm{week} \\
(\text { group }) \\
\text { - anchoring and self-relation techniques [8, 23] }\end{array}$ & $1440 \mathrm{~min}$ \\
- systematic change of time focus (time-line techniques) [6] & $\begin{array}{l}2 \times 60 \mathrm{~min} / \text { week } \\
\text { - ideodynamic techniques [22] }\end{array}$ & $480 \mathrm{~min}$ \\
Jacobson relaxation training & $\begin{array}{l}2 \times 60 \mathrm{~min} / \text { week } \\
(\text { group })\end{array}$ & $480 \mathrm{~min}$ \\
& $\begin{array}{l}1 \times 60 \mathrm{~min} / \mathrm{week} \\
\text { (group) }\end{array}$ & $240 \mathrm{~min}$ \\
Music therapy & & \\
\hline
\end{tabular}

